\title{
Understanding Decision Quality through Satisfaction
}

\author{
João Carneiro ${ }^{1}$, Goreti Marreiros ${ }^{12}$, Paulo Novais ${ }^{3}$, and Ricardo Santos ${ }^{14}$ \\ ${ }^{1}$ GECAD - Knowledge Engineering and Decision Support Group, Porto, Portugal \\ jomrc@isep.ipp.pt \\ ${ }^{2}$ Institute of Engineering - Polytechnic of Porto, Portugal \\ mgt@isep.ipp.pt \\ ${ }^{3}$ University of Minho, Braga, Portugal \\ pjon@di.uminho.pt \\ ${ }^{4}$ CIICESI, School of Technology and Management of Felgueiras - Polytechnic of Porto, Portu- \\ gal \\ rjs@estgf.ipp.pt
}

\begin{abstract}
One of the most important factors to determine the success of an organization is the quality of decisions made. In order to improve the decisions taken and to strengthen the competitiveness of organizations, systems such as Group Decision Support Systems (GDSSs) have been strongly developed and studied in recent decades. The amount of GDSSs incorporating automatic negotiation mechanisms, such as argumentation, is increasing nowadays. The evaluation of these mechanisms and the understanding of their real benefits for the organizations is still a hard challenge. In this article, we propose a model that allows a GDSS to measure the participant's satisfaction with the decision, considering aspects such as problem evaluation, personality, emotions and expectations. This model is intended to enable the understanding of the decision's quality achieved with an argumentation system and to evaluate its capability to potentiate the decision's quality. The proposed model validates all the assumptions found in the literature regarding the participant's satisfaction.
\end{abstract}

Keywords: Decision Satisfaction, Group Decision Support Systems, Outcomes, Affective Computing, Automatic Negotiation, Argumentation

\section{Introduction}

Nowadays the decisions made by managers and executives are mostly performed in groups. Thereby, group decision-making is a process in which a group of people, called participants, act collectively analyzing a set of variables, considering and evaluating the available alternatives in order to select one or more solutions. The number of participants involved in the process is variable and all of them may be either at the same place at the same time or geographically dispersed at different times [6].

It is a known fact that the amount of hours a decision-maker spends in a meeting is not mostly used to make decisions. The time spent on things like social issues, are responsible for consuming the majority of the time of a process [7][8][9]. 
Aiming to improve the decision quality and to facilitate its making in certain scenarios GDSSs have been subject to studies in the last decades. One of the great problems associated to the use of GDSSs is the difficulty to understand the decision makers' satisfaction with the decision made, problem that also exists in decision processes that do not use a GDSS. Being satisfaction a strong indicator of the taken decision quality in the perspective of each participant, its study is very relevant. Higgins [10] says that "a good decision has high outcome benefits (it is worthwhile) and low outcome costs (it is worth it)", and that "independent of outcomes or value from worth, people experience a regulatory fit when they use goal pursuit means that fit their regulatory orientation, and this regulatory fit increases the value of what they are doing". With this, it is possible to understand that the decision quality in the perspective of each participant is related to what he considers relevant. Satisfaction is therefore a strong indicator, not only of the results, but also of the whole decision process. When someone is questioned about the quality of a decision, the answer does not reflect only the assessment of outcomes, but also, even unconsciously; it includes the evaluation process necessary to reach the decision. To understand how suitable a decision is, it is necessary to understand and analyze the means to reach that decision [1][5]. Thus, one should be given prominence to the process, when drawing conclusions about the results.

There is a great variety of factors responsible for affecting the satisfaction of a decision-making element with the decision made in a meeting: emotional variables (affective components) [11][12][13], the process [14][15], the outcomes [10], the factors that affect the situation [16] and expectations [17][18].

Briggs et al. [19] presented a theory of meeting satisfaction, which explains the causes of conflicting research results on meeting satisfaction, as these results have never been fully explained in the Group Support Systems literature. The authors proposed and tested the Satisfaction Attainment Theory (SAT) - a causal model of meeting satisfaction.

Tian et al. [20] conducted a study on how to measure satisfaction based on the emotional space. The results of satisfaction obtained sought to understand the users' acceptance for a product by testing usability.

In their work, Souren et al. [21] explore how the performance of a GDSS affects the different dimensions of satisfaction. They focus on three indicators of group performance, namely: the decision time, the efficiency in decision-making and the number of iterations in the group decision-making process.

The goal of this paper is enable the understanding of the decision's quality achieved with an argumentation system and to evaluate its capability to potentiate the decision's quality. Aiming to contemplate different approaches from researchers of a wide range of areas in this thematic (computer sciences, psychology, economy, etc.), a theoretical-based model is presented, seeking to include in the satisfaction analysis all the necessary variables.

The rest of the paper is organized as follows: the next section is discussed the decision satisfaction thematic and how satisfaction emerges and is related in a decision environment. Section 3 presents the proposed model. Section 4 discusses the relationship between all the points that compose the model and how they measure the partici- 
pant's satisfaction with the decision. Finally, some conclusions are taken in section 5, along with the work to be done hereafter.

\section{Decision Satisfaction}

The satisfaction with a decision resulting from a decision process is something that needs a complex analysis and involves multiple variables. Obviously the satisfaction is related to what we think a good decision is. But what is a good decision? As previously referred, in the common sense a decision is considered good because of the analogy made with the obtained results.

Assumption 1: Decision satisfaction is related with the decision results.

However, psychologically, the results are not enough to make a participant consider a decision as good. Higgins [10] says that "psychologically, then, a decision is perceived as good when its expected value or utility of outcomes is judged to be more beneficial than the alternatives."

Assumption 2: Evaluation of each alternative and comparison between them influences satisfaction.

"The costs of attaining the outcomes can also influence whether a decision is perceived as good. The outcome benefits have to be weighed against the costs of attaining the outcomes. The costs include not only the goods or services one must give in exchange for receiving the benefits but also the costs of the decision-making process itself. The decision-making process that would optimize outcomes might not be used because the costs in cognitive effort or time are too high”.

Assumption 3: The process necessary to reach a decision influences satisfaction.

Therefore, it is clear that there is much more than knowing whether the chosen alternative was the participant's favorite in order to evaluate his satisfaction with the decision. It has been suggested that a purely cognitive approach may be inadequate in modeling satisfaction ratings, so it is particularly important to include emotional variables [11][12][13]. The research that has been made in the field of satisfaction has recognized that there is a need to incorporate the emotional and affective components in regulating consumer's satisfaction [22].

Assumption 4: Emotional and affective components should be included to understand real satisfaction with the decision.

Therefore, it is not only the final results or the decisions made that determine the quality and the satisfaction of the decision. In his work, Higgins says: "We are all familiar with the idea expressed in the maxim of the late-19th-century British states- 
man John Morley, "It is not enough to do good; one must do it the right way," or the coaching classic, "What counts is not whether you win or lose but how you play the game." Such maxims reflect a moral position: Achievements should be evaluated not only in terms of outcomes but also in terms of the means by which they were attained. "The ends do not justify the means."'”[10]. Using the reasoning present in this approach and the moral objective of these famous maxims, the relevance of the process in performing a certain action is easily understood. We can also conclude that the impact of the decision-making process can drastically change the participant's satisfaction regardless of the results.

Consciously or not, people create expectations on (almost) everything. The relationship between expectations and the satisfaction is rather obvious. According to assimilation theory [17], consumers experience a psychological conflict if they perceive a discrepancy between their expectations and their perception of the consumption experience [18]. Moreover, the nature of the expectation-satisfaction relationship may depend on several contextual and behavioral factors. So, users' expectations may have a different impact on the satisfaction formation within particular contexts. Expectations may even be more important when they are unambiguous [23], the product performance is ambiguous [24][25] and/or the consumer is well experienced [26].

Assumption 5: Decision makers create expectations. The expectations are created about everything that is undefined or is going to happen (process that leads to a decision and outcomes).

The consideration of several factors is therefore necessary to obtain a correct approach in the satisfaction analysis of a decision-maker regarding the decision made. The studies addressed in this section show the importance of analyzing the whole decision-making process, and the whole set of actions that involve and influence the participant during the process. We also verified that it is necessary to analyze a set of emotional factors in that process, and that emotional changes mean situations that affect the participant. It is obvious that this brings new challenges, such as to better know the participant to better understand the impact of each situation in each kind of person.

\section{$3 \quad$ Proposed Model}

In this section, we explain the proposed model and how all the points of the model are connected. Furthermore, while we are explaining the model we do the bridge between the points of the model and the assumptions defined before.

Knowing the importance of the process in the satisfaction analysis, all the analysis that purely stress the analysis of the results fall down. In addition, to study the process we cannot focus only on a cognitive approach. Bailey and Pearson [16] agreed that satisfaction in a given situation is the sum of one's feelings or attitudes toward a variety of factors affecting that situation. By creating this model we tried to find the points that can help measure satisfaction without the need to use the final question- 
naires the participants usually have to answer. Our goal is to manipulate certain data, which at the end allows the system itself to evaluate the status of the participants' satisfaction with the decision. Therefore, to analyze the participants' satisfaction with the decision it is important to consider the chosen alternative, his expectations related to the decision and to the process, his personality, and his emotional changes.

\subsection{Point 1 - Satisfaction concerning the chosen alternative}

According to the literature the perception of the decisions' quality is related to the advantages that the participant identifies in that alternative, comparing it against the others. Thus, whereas the preferred alternative is the best in the participants' perspective, the distance between the preferred alternative and the chosen one means a loss of the participants' satisfaction regarding the decision. The loss of satisfaction comprises the difference in the assessment made by the participant for each of the alternatives, as well as what the participant did not achieve with the final decision.

There are five different scenarios that may occur in a meeting, affecting the satisfaction differently:

1. The alternative chosen by the decision-makers is the one chosen as the preferred by the participant. At this point, his satisfaction is related to the assessment he makes on this alternative (Do not forget that it may be the preferred one and not being in anyway the alternative he finds brilliant. The preferred alternative may be one that was not even an option to choose from).

2. The participant starts the meeting with a preference of an alternative, he does not change his opinion during the process, but at the end the chosen alternative will always be one he never took into consideration.

3. The participant may start the meeting with a preference on an alternative and later switch to another one. However, the alternative chosen by the decision-makers ends up being the one he initially chose.

4. The participant may start the meeting with a preference on an alternative and later switch it to another one that eventually will be chosen.

5. The participant starts the meeting with a preference on an alternative, he changes his mind during the process, but at the end the chosen alternative will always be one that he never took into consideration.

This first point of the model intends to satisfy the argument presented in assumption number 2. The usual approach in this situation is taking only into consideration the evaluation done by the participant (decision maker) to the alternative chosen by the group, but as we could verify in the literature this isn't enough. The idea of this point is to understand the satisfaction in terms of alternatives evaluation but also to contemplate a little bit of the assumption number 3. First, it is important the participant evaluates all the alternatives so we can "evaluate each alternative and compare them" (assumption 2), second, it is also very important to understand in what terms the evaluation occurred (assumption 3). 


\subsection{Point 2 - Participants' expectations according to the decision and process}

As we verified in assumption 5 is important to know the participants' expectations according to some issues, in order to have a more accurate perception of the satisfaction, so we think it is important to study the participants' expectations on the following topics:

1. Complexity of the meeting: The participant should be questioned about how he thinks the meeting will be held, in order to reflect on whether he thinks it will have many conflicts and if the understanding among the participants will be problematic. And so, the following question can be asked: "Will this meeting be problematic?”

2. Probability of the participant's preferred alternative to be chosen: Understanding the expectations regarding the probability of the participant's preferred alternative to be chosen. "How likely you think your preferred alternative will be chosen?"

These two topics are the ones we consider most relevant for analyzing the expectations due to the impact the process and the results have on the participant, as previously stated. Besides that, these two topics are easier for the participant to classify regarding its expectations.

There are three different types of impact on satisfaction for each suggested topic:

- Positive Impact: When the final results exceed the expectations.

- Negative Impact: When the expectations are higher than the results achieved.

- Without Impact: When the expectations are achieved.

\subsection{Point 3 - Factor concerning the personality}

The personality is a concept that cannot be briefly defined, because it has a different meaning according to some psychologists who study it. Although most of them would agree that the field of personality is the study of how individuals differ from each other, psychologists would differ about the best way to conceptualize these types of differences [27]. The fact that people differ in their ideas and attitudes, makes them react differently to the factors they are exposed to. Recently, satisfaction is being studied regarding the most different scenarios according to the persons' personality. For instance, Shiammack et al. [28] conducted a study on two factors of The Big Five that contribute to life satisfaction: the Neuroticism and the Extraversion. Another study was conducted by Timothy et al. [29], where they tried to establish a correlation between the values of each type of personality of The Big Five and Job satisfaction.

Knowing that the personality of each one of us influences satisfaction, we think it is relevant to take into account the personality on our analytical model of satisfaction. This point also will helps in work better the assumption 4 . 


\subsection{Point 4 - Emotional changes}

Knowing the importance of the decision-making process, and to make conclusions about the participants' satisfaction regarding decision-making, it is necessary to understand what happens during the process. As mentioned before, it is important to include in the satisfaction analysis affective and emotional components [11][12][13][22].

Having said this, we want to include, at this point, the analysis of generated emotions and to know how they can change the participants' mood. There are two important points to be studied:

1. The sum of emotional spaces that exceed positively or negatively the participant's normal state: it is thus possible to measure the emotional cost that the meeting had on the participant;

2. The participant's mood at the end of the meeting.

\section{Measure the Result of the Satisfaction Using the Model}

To measure the output of each one of the points in the model we must define how they are related. It is considered that the first issue of Point 2 (complexity of the meeting) is strongly related to Point 4 (emotional disorders), while the second issue of Point 2 (probability of the participant's preferred alternative to be chosen) is strongly related to Point 1 (satisfaction with the alternative chosen by the group). So the Point 2 (expectations) will not work isolated, but it will influence the results of the other two points.

The expectations will change the values for Point 1 and Point 4 through a particular impact. The impact causes an expectation that is obviously not always the same. Even knowing the impact that causes the expectation is positive, negative or neutral, it is necessary to quantify that impact.

Beyond expectations, Points 3 and 4 will also have an impact on Point 1 . This is because it is considered that the satisfaction about something always gets related to the evaluation made (the choice of the service, product, etc.). After this evaluation, there are other factors, such as expectations and the process, that change satisfaction. Thus, in this case, the Point 1 will be the analysis performed by a human being, while the other points, according to the context, will affect or not (positively or negatively) the satisfaction.

To make this clearer, the following Fig. 1 illustrates the actual process of measuring satisfaction.

Initially, satisfaction is calculated taking into account the alternative chosen by the group (Point 1 ) and the emotional changes (Point 4) with the impacts caused by the expectations. After the values of these two points have been recalculated, the final values for each point are obtained for the calculation of satisfaction. Emotional changes, as well as personality, will also have an impact on the participant's satisfaction with the option chosen by the group. 


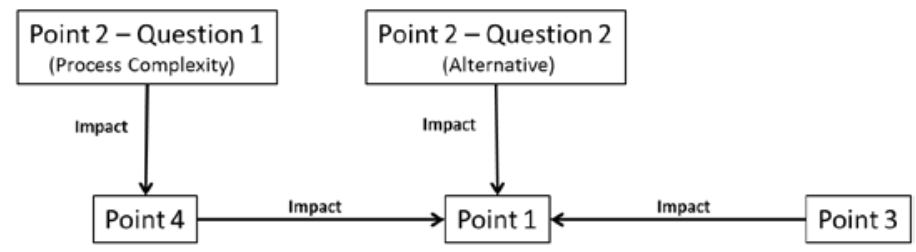

Fig. 1. Impacts caused by each of the points of the model

The use of the personality in the final calculation may not exist directly. This happens for example when we are dealing with a multi-agent system in which the arguments used by the agents are according to the identified personalities. This will generate emotions and the change of mood regarding the personality. Thus, Point 3 is not covered in the final formula despite being covered by the system indirectly.

\section{$5 \quad$ Conclusions and Future Work}

Several concepts of satisfaction and the existing models to assess satisfaction were presented. Furthermore, this paper proposed a whole new model which pretends the assessment of the participants' satisfaction in a meeting, supported by a GDSS. We believe that the proposed model allows the attainment of a large amount of useful and valuable information. The points presented in proposed model try to cover every assumption created after reading the literature on different areas (psychology, computer science, economy and sociology).

This is the first model of satisfaction analysis, which considers every point found as relevant in the literature.

As future work, first we will turn this model mathematical and after we will conduct a case study with real people, in partnership with psychologists. With that work, we also intend to make the model assertive by the possible improvements that might result after analyzing and studying the collected data.

\section{Acknowledgements}

This work is supported by FEDER Funds through the "Programa Operacional Factores de Competitividade - COMPETE” program and by National Funds through FCT "Fundação para a Ciência e a Tecnologia" under the project: FCOMP-01-0124FEDER-PEst-OE/EEI/UI0760/2011 and SFRH/BD/89697/2012.

\section{References}

1. L. R. Beach, Image Theory: Decision Making in Personal and Organizational Contexts, New York: Wiley, 1990

2. J.G. March, Primer on Decision Making: How Decision Happen, New York: Free Press, 1994 
3. F. Luthans, Organizational Behavior. In 10th ed. McGraw Hill Irwin, Boston, 2005

4. C. Argyris and D. Schon, Theory in Practice, Jossey-Bass, San Francisco, 1974

5. L. R. Hoffman, Applying Experimental Research on Group Problem Solving to Organizations, Journal of Applied Behavioral Sciences, 1979, pp. 375-391

6. H. Mintzberg, The Nature of Managerial Work, Harper and Row, New York, 1973

7. E. T. Higgins, Making a Good Decision: Value From Fit, American Psychologist, vol 55, no. 11,2000 , pp. $1217-1230$

8. V. Liljander and T. Strandvik, Emotions in Service Satisfaction, International Journal of Service Industry Management, vol. 8, No. 2, 1997, pp. 148-169

9. R. L. Oliver, R. T. Rust, S. Varki, Customer Delight: Foudations Findings, and Managerial Insight, Journal of Retailing, vol. 73, no. 3, 1997, pp. 311-336

10. J. Wirtz and J. E. G. Bateson, Consumer Satisfaction with Services: Integrating the Environment Perspective in Services Marketing into the Traditional Disconfirmation Paradigm, Journal of Business Research, vol. 44, 1999, pp. 55-66

11. H. A. Simon, A Behavioral Model of Rational Choice, Quarterly Journal of Economics, vol. 69, 1955, pp. 99-118

12. H. A. Simon, Motivational and Emotional Controls of Cognition, Psychological Review, vol. 74, 1967, pp. 29-39

13. J. E. Bailey, S. W. Pearson, Development of a Tool for Measuring and Analysing Computer User Satisfaction, Management Science, vol. 29, no. 5, 1983, pp. 530-545

14. M. Sherif, C. I. Hovland, Social Judgment: Assimilation and Contrast Effects in Communication and Attitude Change, New Have, CT: Yale University Express, 1961

15. I. A. Bosque, S. M. Collado, The Role of Expectations in the Consumer Satisfaction Formation Process: Empirical Evidence in the Travel Agency Sector, Tourism Management, vol 27, no. 3, 2006, pp. 410-419

16. O. Briggs, G. Vreede and B. Reinig, A Theory and Measurement of Meeting Satisfaction, Proc. of the 36th Annual Hawaii International Conference on System Sciences, 2003, pp. 25.3

17. X. Tian, W. Hou, K. Yuan, A Study on the Method of Satisfaction Measurement Based on Emotion Space, Proc. of 9th International Conference on Computer-Aided Industrial Design and Conceptual Design, 2008, pp.39-43

18. P. Souren, S. Priya, K. Ramamurthy, User Satisfaction with System, Decision Process, and Outcome in GDSS Based Meeting: An Experimental Investigation, Proc. of the 37th Annual Hawaii International Conference on System Sciences, 2004, pp.10037.2

19. J. Wirtz, A. S. Mattila and R. L. P. Tan, The Moderating Role of Target-Arousal on the Impact of Affect on Satisfaction - An Examination in the Context of Service Experiences, Journal of Retailing, vol. 76, no. 3, 2000, pp. 347-365

20. P. Nyer, The Determinants of Satisfaction: an Experimental Verification of Moderating role of Ambiguity. Advances in Consumer Research, vol. 24, 1996, pp. 255-259

21. Y. Yi, The Determinants of Consumer Satisfaction: The Moderating Role of Ambiguity. Advances in Consumer Research, vol. 20, 1993, pp. 502-506

22. R. L. Oliver, Satisfaction: A Behavioral Perspective on the Consumer, New York: McGraw-Hill, 1997

23. M. Soderlund, Customer Familiarity and its Effects on Satisfaction and Behavioral Intentions, Psychology \& Marketing, vol. 19, no. 10, 2002, pp. 861-879

24. R. Santos, G. Marreiros, C. Ramos, J. Neves and J. Bulas-Cruz, Personality, Emotion, and Mood in Agent-Based Group Decision Making, IEEE Intelligent Systems, vol. 26, no. 6, 2011, pp. 58-66 
25. U. Shimmack, S. Oishi, R. M. Furr and D. C. Funder, Personality and Life Satisfaction: A Facet- Level Analysis, Personality and Social Psychology Bulletim, vol. 30, no. 8, 2004,pp. 1062-1075

26. A. Timothy, D. Heller, M. K. Mount, Five-Factor Model of Personality and Job Satisfaction, Journal of Applied Psychology, vol. 87, no. 3, 2002, pp. 530-541 